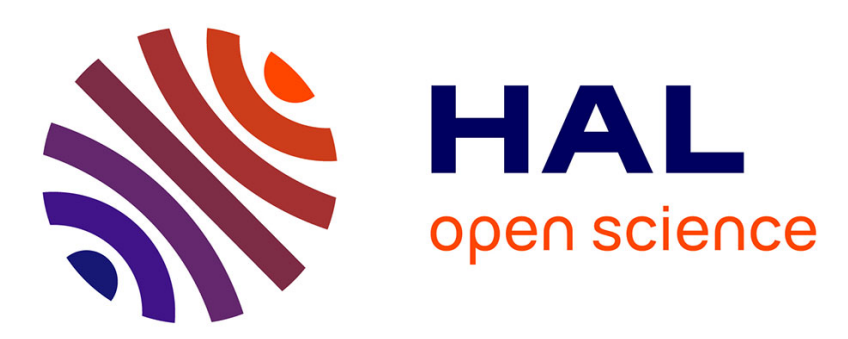

\title{
Atomistic modeling of point defect contributions to swelling in Xe- implanted silicon carbide
}

Laurent Pizzagalli

\section{To cite this version:}

Laurent Pizzagalli. Atomistic modeling of point defect contributions to swelling in Xe- implanted silicon carbide. Journal of Nuclear Materials, 2018, 512, pp.349-356. 10.1016/j.jnucmat.2018.10.024 . hal-02418257

\section{HAL Id: hal-02418257 \\ https://hal.science/hal-02418257}

Submitted on 20 Dec 2019

HAL is a multi-disciplinary open access archive for the deposit and dissemination of scientific research documents, whether they are published or not. The documents may come from teaching and research institutions in France or abroad, or from public or private research centers.
L'archive ouverte pluridisciplinaire HAL, est destinée au dépôt et à la diffusion de documents scientifiques de niveau recherche, publiés ou non, émanant des établissements d'enseignement et de recherche français ou étrangers, des laboratoires publics ou privés. 


\title{
Atomistic modeling of point defect contributions to swelling in Xe-implanted silicon carbide
}

\author{
L. Pizzagalli ${ }^{\mathrm{a}}$ \\ ${ }^{a}$ Institut P', CNRS UPR 3346, Université de Poitiers, SP2MI, Boulevard Marie et Pierre Curie, TSA 41123, 86073 Poitiers Cedex 9, France
}

\begin{abstract}
Atomistic calculations using a newly developed $\mathrm{Xe}-\mathrm{SiC}$ interatomic potential are carried out to determine the contributions of point defects to the strain build up in Xe implanted $4 \mathrm{H}-\mathrm{SiC}$. Relaxation volumes for individual point defects are calculated, and analysed in comparison to the swelling determined in a simulation including a large homogeneous distribution of these defects. These investigations confirm the negligible influence of the implanted gas, with the swelling mostly originating from intrinsic point defects generated by implantation. Using experimental swelling data, possible point defects distributions are determined as a function of the dose. Strain reduction during annealing simulations is shown to be due to dynamic recombination of point defects, with an estimated activation energy in excellent agreement with experiments.
\end{abstract}

Keywords: SiC, Point defects, Strain, Atomistic calculations

\section{Introduction}

Silicon carbide $\mathrm{SiC}$ is an appealing material for various applications due to its excellent properties $[1,2]$. In particular, $\mathrm{SiC}$ is a very strong and hard material, and can tolerate harsh environmental conditions such as high temperature or corrosion. $\mathrm{SiC}$ is also characterized by a high resistance to irradiation, with a good potential for various applications in a nuclear context. For instance, $\mathrm{SiC}$ is used in cladding barriers for fuel, with the aim of retaining fission products such as He or Xe. In future fusion reactors, $\mathrm{SiC}$ is envisioned for use as a structural material [3, 4].

A critical and general issue associated with fuel cladding is the accumulation of damage due to continuous irradiation, that ultimately leads to degradation of the material. In the case of $\mathrm{SiC}$, irradiation at low temperature, i.e. lower than $250{ }^{\circ} \mathrm{C}$, leads to amorphization [5-7]. At higher temperatures, amorphization is prevented by dynamic recovery. However, damage accumulation still occurs, and produces a strain build up in the irradiated regions leading to an observable swelling. Since $\mathrm{SiC}$ is inherently brittle, such strains can lead to cracks, what is a dramatic issue for a confining material. A significant strain relaxation can be obtained when high temperature annealing experiments are carried out. Previous investigations of Heimplanted $\mathrm{SiC}$ suggest that this recovery mechanism is mainly related to point defects migration $[8,9]$.

Recently, Jiang et al. have investigated the properties of $4 \mathrm{H}-\mathrm{SiC}$ monocrystals following the implantation of heavy noble gas atoms Xe and Ar [10]. In particular they measured by $\mathrm{X}$-ray diffraction the swelling due to the damage in the implanted layer as a function of the fluence. The authors con-

\footnotetext{
Email address: Laurent.Pizzagalli@univ-poitiers.fr (L. Pizzagalli)
}

cluded that the lattice strain build up is mainly associated with interstitial defects created during implantation, while the noble gas atoms contribution is negligible. In order to confirm this point, it would be necessary to determine the contribution of all defects created by irradiation. Such a feat is difficult to achieve experimentally, but atomistic simulations offer a possible alternative.

To my knowledge, little theoretical information is available regarding the strain build up associated to single point defects in silicon carbide associated to noble gas implantation. Furthermore, potential contributions to swelling from xenon atoms, in interaction or not with defects, are not documented. To fill this gap, atomistic simulations have been carried out and reported in the present paper. The next section describes the development of the $\mathrm{Xe}-\mathrm{Si}-\mathrm{C}$ interatomic potential which is used in these simulations. Section 3 summarizes results on the computed relaxation volume for single point defects. In section 4 are described molecular dynamics calculations of large systems encompassing variable amount of defects, compared to experiments in Ref. [10]. The last section focus on the investigation of dynamic recovery during annealing simulations.

\section{2. $\mathrm{Xe}-\mathrm{Si}-\mathrm{C}$ potential}

There are several available interatomic potentials for silicon carbide (to cite a few [11-14]). Among these, the one proposed by Erhart and Albe is interesting since it accurately reproduces elastic properties [13], which is a critical aspect for lattice deformation. To describe $\mathrm{Xe}$ in $\mathrm{SiC}$, suitable interatomic potential functions between $\mathrm{Xe}$ and $\mathrm{Si} / \mathrm{C}$ are also required. Unfortunately, it seems that none is available in the literature.

The first task is then to develop potential functions for Xe-Si and $\mathrm{Xe}-\mathrm{C}$ interactions. The Buckingham pair potential is used 
Table 1: Calculated energies (in $\mathrm{eV}$ ) required to insert a single Xe atom into various defect configurations in cubic silicon carbide: in interstitial positions (tetrahedral $\mathrm{Si}, \mathrm{C}$, and bond-centered sites), in mono-vacancies ( $\mathrm{Si}, \mathrm{C}$ ), in a carbon vacancy next to a carbon antisite, in di-vacancies ( $\mathrm{Si}-\mathrm{C}, \mathrm{Si}-\mathrm{Si}$ and $\mathrm{C}$ $\mathrm{C}$ ), in tri-vacancies ( $\mathrm{Si}-\mathrm{C}-\mathrm{Si}$ and $\mathrm{C}-\mathrm{Si}-\mathrm{C}$ ), and in a quadri-vacancy (compact squared structure). * indicate configurations used to fit the $\mathrm{Xe}-\mathrm{C}$ and $\mathrm{Xe}-\mathrm{Si}$ potential functions. The values obtained in this work ( $4^{\text {th }}$ column $)$ are compared to available density functional theory results ( $2^{\text {nd }}$ and $3^{\text {rd }}$ columns).

\begin{tabular}{cccc}
\hline \hline Conf. & {$[15]$} & {$[16]$} & This work \\
\hline $\mathrm{I}\left(\mathrm{T}_{\mathrm{Si}}\right)$ & 25.8 & 24.00 & 20.55 \\
$\mathrm{I}\left(\mathrm{T}_{\mathrm{C}}\right)$ & 25.7 & 23.21 & 18.41 \\
$\mathrm{I}(\mathrm{BC})$ & $18.1-18.5$ & 18.49 & 19.17 \\
$\mathrm{~V}_{\mathrm{Si}} *$ & 6.5 & 6.41 & 5.42 \\
$\mathrm{~V}_{\mathrm{C}} *$ & 9.2 & 8.15 & 8.65 \\
$\mathrm{~V}_{\mathrm{C}}+\mathrm{C}_{\mathrm{Si}} *$ & 7.9 & & 7.63 \\
$\mathrm{~V}_{\mathrm{C}} \mathrm{V}_{\mathrm{Si}} *$ & 4.6 & 4.33 & 2.71 \\
$\mathrm{~V}_{\mathrm{Si}} \mathrm{V}_{\mathrm{Si}} *$ & 4.5 & & 6.43 \\
$\mathrm{~V}_{\mathrm{C}} \mathrm{V}_{\mathrm{C}} *$ & 7.9 & & 9.15 \\
$\mathrm{~V}_{\mathrm{Si}} \mathrm{V}_{\mathrm{C}} \mathrm{V}_{\mathrm{Si}}$ & 2.4 & & 1.87 \\
$\mathrm{~V}_{\mathrm{C}} \mathrm{V}_{\mathrm{Si}} \mathrm{V}_{\mathrm{C}}$ & 5.6 & & 1.81 \\
$\mathrm{~V}_{\mathrm{C}} \mathrm{V}_{\mathrm{Si}} \mathrm{V}_{\mathrm{C}} \mathrm{V}_{\mathrm{Si}}$ & 3.0 & & 2.75 \\
\hline \hline
\end{tabular}

Table 2: Fitted parameters for the $\mathrm{Xe}-\mathrm{C}$ and $\mathrm{Xe}-\mathrm{Si}$ Buckingham pair functions, with the last parameter $C=0$ in eq. (1).

\begin{tabular}{ccc}
\hline \hline & $A(\mathrm{eV})$ & $\rho(\AA)$ \\
\hline $\mathrm{Xe}-\mathrm{C}$ & 18030 & 0.2468 \\
$\mathrm{Xe}-\mathrm{Si}$ & 8935 & 0.2589 \\
\hline \hline
\end{tabular}

as a starting function:

$$
V(r)=A \exp (-r / \rho)-\frac{C}{r^{6}}
$$

$r$ being the atomic separation. The three parameters $A, \rho$, and $C$ are fitted to reproduce first-principles calculations of excess energies associated with the insertion of a xenon atom in different locations (Table 1). I find that the second term in Eq. (1) is negligible and has no influence on the fitting process, and $C$ is set to 0 for both $\mathrm{Xe}-\mathrm{C}$ and $\mathrm{Xe}-\mathrm{Si}$ functions. The best set of parameters is reported in Table 2.

In a second step, the pair functions are modified at short separations to match a Ziegler-Biersack-Littmark (ZBL) pair potential [17]. The latter is more meaningful in this regime which could be useful for future uses of this potential, to describe highly energetic Xe atoms in interaction with $\mathrm{SiC}$ for instance. The two potentials are linked by a $5^{\text {th }}$ order polynomial, in the range 1.7-2.05 $\AA$ for $\mathrm{Xe}-\mathrm{Si}$, and 2.3-2.6 $\AA$ for $\mathrm{Xe}-\mathrm{C}$, allowing for continuous first and second derivatives. The generated functions are then tabulated.

Finally, the Xe-Xe interaction is described by the HartreeFock-Dispersion HFD-B2 potential as proposed by Dham et al. [18]:
Table 3: Relaxation volumes $\left(\AA^{3}\right)$ computed at $300 \mathrm{~K}$ and swelling $\left(\times 10^{-5}\right)$ as defined in equation (6).

\begin{tabular}{cccc}
\hline \hline & $\Omega\left(\AA^{3}\right)$ & $\omega\left(\AA^{3}\right)$ & $s\left(\times 10^{-5}\right)$ \\
\hline $\mathrm{V}_{\mathrm{Si}}$ & 4.96 & 2.10 & 0.2010 \\
$\mathrm{~V}_{\mathrm{C}}$ & 6.37 & 2.25 & 0.2154 \\
$\mathrm{C}_{\mathrm{Si}}$ & -9.73 & -4.07 & -0.3896 \\
$\mathrm{Si}_{\mathrm{C}}$ & 23.16 & 9.18 & 0.8787 \\
$\mathrm{I}_{\mathrm{Si}}$ & 48.60 & 18.88 & 1.8072 \\
$\mathrm{I}_{\mathrm{C}}$ & 20.01 & 8.13 & 0.7782 \\
$\mathrm{Xe}$ in $\mathrm{V}_{\mathrm{Si}}$ & 20.66 & 8.64 & 0.8270 \\
$\mathrm{Xe}$ in $\mathrm{V}_{\mathrm{C}}$ & 45.16 & 18.30 & 1.7517 \\
$\mathrm{I}_{\mathrm{Xe}}$ & 36.99 & 14.21 & 1.3602 \\
\hline \hline
\end{tabular}

$$
\begin{aligned}
V(r) & =\varepsilon V^{*}(r) \\
V^{*}(r) & =A^{*} \exp \left(-\alpha^{*} r+\beta^{*} r^{2}\right)-F(r) \sum_{j=0}^{2} \frac{c_{2 j+6}}{r^{2 j+6}}
\end{aligned}
$$

with

$$
\begin{aligned}
F(x) & =\exp \left[-\left(\frac{D}{r}-1\right)^{2}\right], r<D \\
& =1, \quad r \geq D
\end{aligned}
$$

At short range, this potential is also replaced by the ZBL potential, with a smooth connection by a $5^{\text {th }}$ order polynomial in the range 1.15-1.25 $\AA$. Again, the resulting final pair function is tabulated.

Table 1 provides an overview of the capabilities of the Xe$\mathrm{SiC}$ potential to model xenon atoms in interaction with vacancy defects in cubic SiC. The reported energy calculations have been performed using the code LAMMPS [19], in the same conditions than the first-principles simulations (i.e. in small supercells) for a meaningful comparison. The agreement with the reference configurations used in the fit is fair. In particular, the excess energy to insert a Xe atom in a Si or C monovacancy is well reproduced. The potential yields underestimated values for $\mathrm{V}_{\mathrm{C}} \mathrm{V}_{\mathrm{Si}}$, and overestimated ones for $\mathrm{V}_{\mathrm{Si}} \mathrm{V}_{\mathrm{Si}}$ and $\mathrm{V}_{\mathrm{C}} \mathrm{V}_{\mathrm{C}}$. The potential also yields slightly too low excess energies in case of trivacancies, but correctly models a Xe in a quadrivacancy.

Furthermore, I also tested the accuracy of the potential to model interstitial xenon atoms, in tetrahedral and bond-centered configurations. Very high excess energies are obtained (Table 1), in agreement with reference first-principles calculations, although the tetrahedral configurations energies are probably a bit too low.

\section{Relaxation volume of single defects}

The aforementioned potential is then used to compute the relaxation volume at $300 \mathrm{~K}$ associated with different point defects in $4 \mathrm{H}-\mathrm{SiC}$. Each defect configuration is embedded in a 
supercell of dimensions $6 \mathrm{a}_{0} \times 4 \sqrt{3} \mathrm{a}_{0} \times 2 \mathrm{c}_{0}$ (768 atoms). I find $\mathrm{a}_{0}(300 \mathrm{~K})=3.0883 \AA$ and $\mathrm{c}_{0}(300 \mathrm{~K})=10.0865 \AA$, i.e. the reference volume of the supercell at $300 \mathrm{~K}$ is $7997.998 \AA^{3}$. Molecular dynamics simulations in the NPT ensemble are performed at $300 \mathrm{~K}$ and zero pressure during $10 \mathrm{ps}$ for each case. Once the system is thermalized, the supercell volume is calculated as an average, and the relaxation volume obtained by subtracting the reference volume. It is important to emphasize that the relaxation volume does not depend on the supercell size, except for the elastic interaction between the defect and its periodically replicated images. Since the latter varies as $1 / r^{3}$ for point defects, one expect converged values for moderately sized supercell. Tests have been performed using different supercell dimensions to confirm this point.

I first consider single $\mathrm{Si}$ and $\mathrm{C}$ interstitials, since these have been shown to constitute most of the created defects during cascades [20]. There are many possible stable interstitial configurations, with the extra atom being located in tetrahedral sites or forming different kinds of dumbbells with lattice atoms [21]. Besides, not all lattice atoms are equivalent in $4 \mathrm{H}-\mathrm{SiC}$, what further increases the number of configurations to investigate. It is not clear which of these configurations will be dominant in the context of implantation. Also, the empirical potential is not able to fully reproduce the defects energetics ordering compared to first-principles calculations [13]. To circumvent these issues, ten different defect configurations are generated for the $\mathrm{Si}(\mathrm{C})$ interstitial according to the following procedure: a lattice $\mathrm{Si}$ or $\mathrm{C}$ atom is first randomly selected; then random coordinates are generated for the interstitial atom with the requirement that the minimal (maximal) distance along a direction with the lattice atom is $0.3 \AA(1.1 \AA)$. The structure analysis at the end of the 10 ps run reveals various geometries, e.g. with $\mathrm{Si} / \mathrm{C}$ in dumbbells and tetrahedral configurations. The relaxation volume for $\mathrm{Si} / \mathrm{C}$ interstitial is then obtained as an average of all cases. A similar method is used for the xenon interstitial. For the remaining defects, i.e. $\mathrm{Si} / \mathrm{C}$ vacancies and antisites, and $\mathrm{Xe}$ atom located in $\mathrm{Si} / \mathrm{C}$ vacancies, the average is performed taking into account inequivalent $4 \mathrm{H}$ lattice sites.

The calculated relaxation volumes are reported in the table 3. Low but positive values are obtained for $\mathrm{Si}$ and $\mathrm{C}$ vacancies, in agreement with previous findings of an outward relaxation in the vicinity of vacancies [22]. For the antisites, a negative relaxation volumes is found for $\mathrm{C}_{\mathrm{Si}}$, and a positive one for $\mathrm{Si}_{\mathrm{C}}$, also in agreement with earlier first principles calculations [22]. These results can be understood on the basis of simple physical arguments, a $\mathrm{C}_{\mathrm{Si}}$ antisite implying the formation of stretched $\mathrm{C}-\mathrm{C}$ bonds, while a $\mathrm{Si}_{\mathrm{C}}$ antisite leads to compressed $\mathrm{Si}-\mathrm{Si}$ bonds. Large relaxation volumes are obtained for Si interstitials, and to a lesser extent for $\mathrm{C}$ interstitials. To my knowledge, no reference values, obtained with first principles calculations for instance, are available. It is then difficult to estimate whether these values are realistic or overestimated, because of the potential for instance. Nevertheless, my results are in fair agreement with an earlier investigations based on the Tersoff potential [23].

Positive relaxation volumes, i.e. supercell expansion, are always obtained for Xe interstitials or in vacancies, since Xe-
$\mathrm{Si}$ and $\mathrm{Xe}-\mathrm{C}$ repulsive interactions start at separations greater than the $\mathrm{Si}-\mathrm{C}$ bulk distance. Surprisingly, the largest relaxation volume corresponds to $\mathrm{Xe}$ in a $\mathrm{C}$ vacancy, while a $\mathrm{Xe}$ interstitial leads to a slightly lower value. The analysis of the investigated Xe interstitial structures reveals that configurations with the largest proportion of $\mathrm{Xe}-\mathrm{Si}(\mathrm{Xe}-\mathrm{C})$ first neighbors are associated with the largest (lowest) relaxation volumes, respectively. This explains why the $\mathrm{Xe}$ interstitial relaxation volume is lower in average than $\mathrm{Xe}$ in a $\mathrm{C}$ vacancy.

The relaxation volume as reported above is not directly related to the swelling observed in Xe-implanted SiC samples. In fact the latter occurs only along the implantation axis, here [0001], while along the two remaining axis, the dimensions are necessarily constrained to bulk values. This is the same distinction between true and normal strain as reported in the literature [24]. I define a second relaxation volume, $\omega$, corresponding to the volume expansion along the $\vec{c}$ axis only, during relaxation. Determining $\omega$ from $\Omega$ using elasticity theory is possible [25], but can be inaccurate due to the anisotropic deformation of most $4 \mathrm{H}-\mathrm{SiC}$ point defects. Instead here $\omega$ is computed by performing additional molecular dynamics calculations, for which volume expansion is only possible along the $\vec{c}$ axis (the two other dimensions are fixed using $\mathrm{a}_{0}(300 \mathrm{~K})=3.0883 \AA$ ). Like for $\Omega, \omega$ does not depend on the supercell size. The results, reported in Table 3, follow qualitatively the same trends than for $\Omega$. In all cases, the ratio between $\Omega$ and $\omega$ is also close to the constant value predicted by elasticity theory $[24,25]$.

Each point defect resulting from the implantation process will contribute to the swelling of the material. The number of defects of type $\alpha$ in a volume $V$ is given by $N_{\alpha}=C_{\alpha} V$, with $C_{\alpha}$ the concentration. Assuming that the interaction between defects is negligible, the volume expansion associated to swelling is given by $\delta V=\sum_{\alpha} N_{\alpha} \omega_{\alpha}=V \sum_{\alpha} C_{\alpha} \omega_{\alpha}$. However, one could expect interactions between the generated point defects, and also aggregation, and this expression should only be used as an estimate. Nevertheless, the critical issue is not this one, but rather the unknown concentration of point defects in the implanted materials. A software like SRIM [26] predicts the amount of lattice atoms displaced by the impinging ions, but dynamic recombination is not taken into account, and the resulting point defects concentrations are unrealistically high. As an illustration, let us consider the case of a $540 \mathrm{keV}$ xenon implantation in $4 \mathrm{H}-\mathrm{SiC}[10]$. For a fluence of $1 \times 10^{14} \mathrm{Xe} . \mathrm{cm}^{-2}$, the damage distribution maximum corresponds to about $1 / 3$ of lattice atoms being displaced. Not only such a proportion of interstitials and vacancies would obviously destabilize the crystal lattice, but should also produce an improbable huge swelling. In fact the volume expansion would be about $50 \%$ using the relaxation volume values reported in Table 3. In the following section, I propose an alternative strategy to tackle this issue.

\section{Swelling of large implanted systems}

The influence of point defects on the swelling of $4 \mathrm{H}-\mathrm{SiC}$ is next studied by performing molecular dynamics simulations of large $4 \mathrm{H}-\mathrm{SiC}$ systems. The new supercell dimensions are now $33 \mathrm{a}_{0} \times 19 \sqrt{3} \mathrm{a}_{0} \times 10 \mathrm{c}_{0}(100320$ atoms $)$. This corresponds to $\mathrm{a}$ 


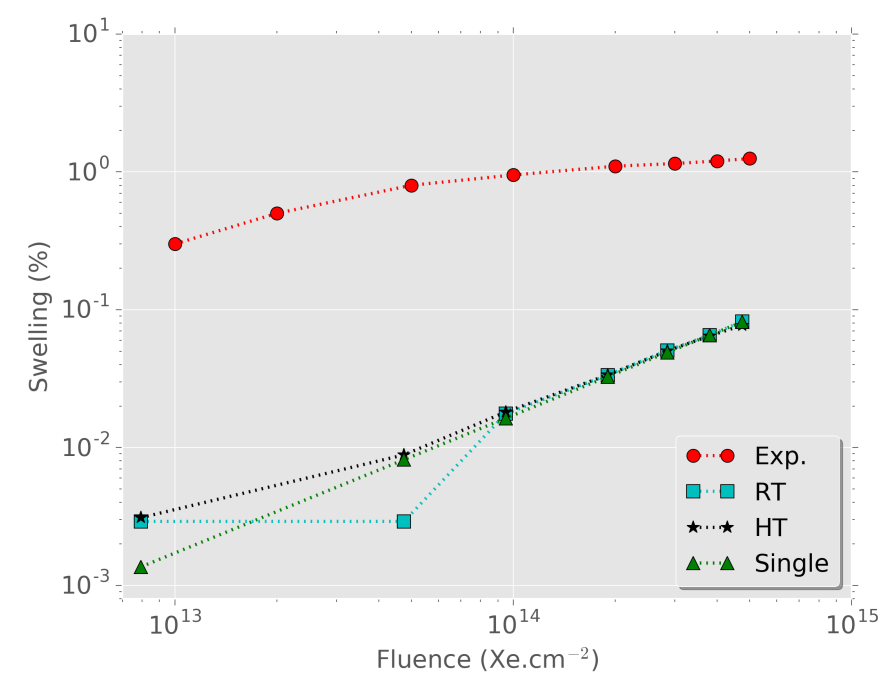

Figure 1: Local swelling (in \%) as a function of the fluence from experiments [10] (red circles), from simulations with Xe atoms only (RT (blue squares) and HT (black stars) setups), and evaluated from the single defect relaxation volume in Table 3 (green triangles).

volume of $1044.691 \mathrm{~nm}^{3}$ using the $300 \mathrm{~K}$ lattice constants. The targeted experiment is a $540 \mathrm{keV} \mathrm{Xe}$ implantation with a fluence ranging from $1 \times 10^{13} \mathrm{Xe} . \mathrm{cm}^{-2}$ to $5 \times 10^{14} \mathrm{Xe} . \mathrm{cm}^{-2}$, as reported in [10]. SRIM calculations predict that the implanted Xe distribution maximum occurs at a depth of $150 \mathrm{~nm}$ and ranges from $1.21 \times 10^{18} \mathrm{Xe} . \mathrm{cm}^{-3}$ to $6.04 \times 10^{19} \mathrm{Xe} . \mathrm{cm}^{-3}$ for these fluences. In the supercell considered here, it corresponds to a number of implanted Xe atoms from 1 to 63 . The predicted maximum damage is reached at a depth of about $110 \mathrm{~nm}$. As explained in the previous section, SRIM calculations cannot be used to set vacancies and interstitials quantities in the simulations. Instead, I consider variable amounts of created $\mathrm{Si}$ and $\mathrm{C}$ Frenkel pairs (FP) in our supercell: from 200 to 1000 for Si (i.e. concentrations in the range $1.91-9.57 \times 10^{20} \mathrm{FP}^{-\mathrm{cm}^{-3}}$ ), and from 200 to 1800 for $\mathrm{C}\left(0.191-1.72 \times 10^{21} \mathrm{FP}_{\mathrm{cm}} \mathrm{cm}^{-3}\right)$. A larger maximum number of $\mathrm{C} \mathrm{FP}$ is considered because it is easier to displace atoms in the $\mathrm{C}$ sublattice than in the Si sublattice [27].

To generate the input configurations, the xenon atoms are inserted in the $4 \mathrm{H}$ lattice according to the same procedure than for single defects, described in the previous section. Si and C FP are also generated with the same scheme, the atoms removed to create a vacancy being positioned in a random interstitial configuration. For each cases, a short molecular dynamics simulation (0.1 ps) is first performed in the NVE ensemble, with the additional constraint that the atomic displacements at each integration step are at most $0.01 \AA$. This stage allows for preventing potential situations where two atoms are initially very close. Next, two different setups are used. In the first one (hereinafter called RT), a 10 ps run is performed at $300 \mathrm{~K}$, during which the system can expand only along $\vec{c}$. The idea is to determine the swelling associated with the initial defects distribution, with limited recombination or aggregation. In the second setup (HT), a NVT run is performed at $2500 \mathrm{~K}$ for $10 \mathrm{ps}$, then the temperature is linearly decreased to $300 \mathrm{~K}$ for $5 \mathrm{ps}$. Fi-

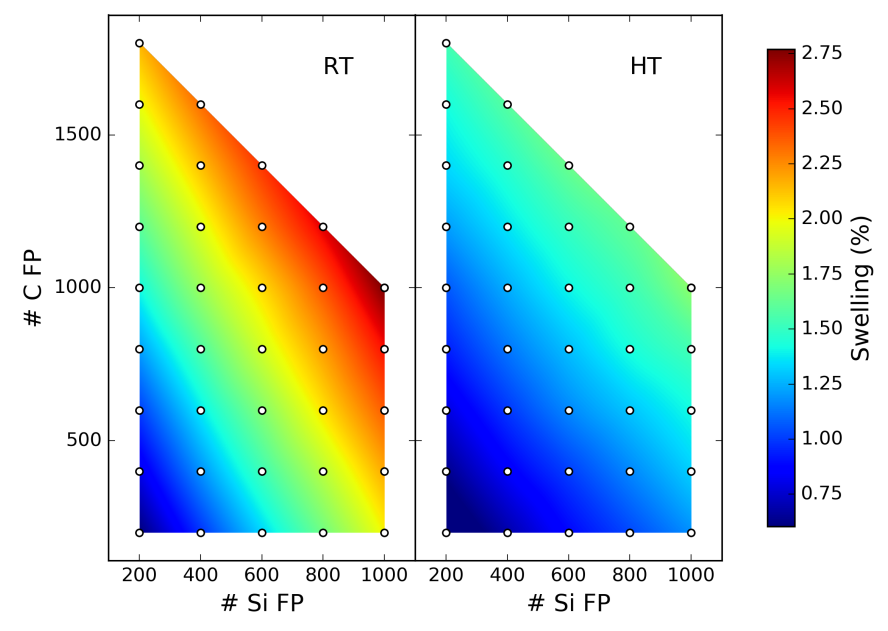

Figure 2: Swelling (in \%) as a function of the number of Si and C Frenkel pairs in the 100320-atoms system, for both RT and HT setups. The circles represent the calculated configurations, and the full swelling map is obtained by cubic interpolation.

nally, a 5 ps run is performed at $300 \mathrm{~K}$ with expansion allowed along $\vec{c}$. The short high temperature should promote a limited vacancy-interstitial recombination and the potential formation of more stable defect complexes. In both cases, each run is ended by a complete forces relaxation in a supercell of fixed dimensions, thus removing any thermal displacements and allowing for a better identification of the defects configurations.

The role of xenon atoms on the swelling is first examined. Simulations with an increasing amount of xenon atoms (up to 60) but with no Si and C FP, are performed and the resulting swelling is compared to experimental data in Figure 1. For both RT and HT simulations, it appears that the swelling due to xenon atoms is only about $1 \%$ of the measured swelling at the lowest fluence $\left(1 \times 10^{13} \mathrm{Xe} . \mathrm{cm}^{-2}\right)$, and increases up to $6.5 \%$ at the highest one $\left(5 \times 10^{14} \mathrm{Xe} . \mathrm{cm}^{-2}\right)$. The experimentally observed swelling is then mainly due to point defects created during the xenon implantation, which confirms previous conclusions [10]. I also try to estimate the swelling from the relaxation volume $\omega$ of single defects calculated in the previous section, assuming an independent contribution of each Xe atom. For a given defect $\alpha$, the latter is obtained with the relation:

$$
s_{\alpha}=\frac{z_{\alpha}-z_{0}}{z_{0}}=\frac{\omega_{\alpha}}{x_{0} y_{0} z_{0}}
$$

since $\omega_{\alpha}=x_{0} y_{0}\left(z_{\alpha}-z_{0}\right),\left(x_{0}, y_{0}\right.$, and $z_{0}$ are the supercell dimensions with no defects, and $z_{\alpha}$ is the dimension along $\vec{c}$ of the supercell containing the defect $\alpha$ ). $s_{\alpha}$ for each kind of defects are reported in Table 3 . Figure 1 shows that this approach yields swelling values in excellent agreement with other calculations, and provide a suitable alternative to direct simulations.

I now focus on the effect of intrinsic point defects on the swelling. Figure 2 represents the swelling from RT and HT simulations, for different proportions of Si and C FP, but with no xenon atoms. A first straightforward observation is that the chosen amounts of Si and C FP are appropriate, since the experimental swelling measurements are in the range of the cal- 


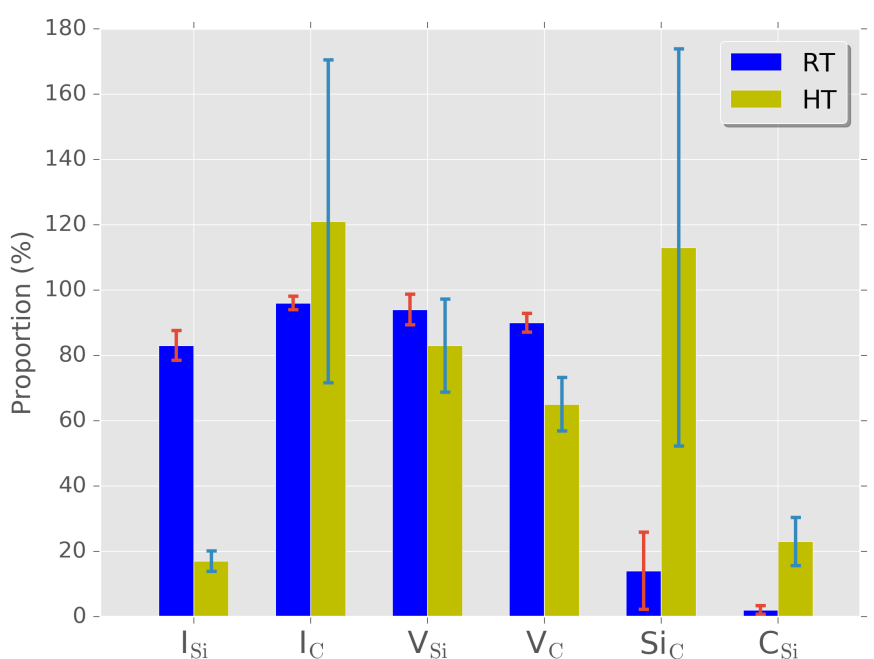

Figure 3: Point defects distribution at the end of RT and HT simulations. The bars represent the average numbers relative to the initial amounts of $\mathrm{Si} \mathrm{FP}$ (for $\mathrm{I}_{\mathrm{Si}}, \mathrm{V}_{\mathrm{Si}}$, and $\mathrm{Si}_{\mathrm{C}}$ ) and of $\mathrm{C} \mathrm{FP}$ (for $\mathrm{I}_{\mathrm{C}}, \mathrm{V}_{\mathrm{C}}$, and $\mathrm{C}_{\mathrm{Si}}$ ). Standard deviations are shown as colored lines on top of the bars.

culated ones. Also, it is clear that the swelling increases as a function of $\mathrm{C}$ and Si FP numbers, and that defects created on the Si sub-lattice have a larger contribution to the swelling than those on the $\mathrm{C}$ sub-lattice. This is not surprising given the largest relaxation volumes $\omega$ for $\mathrm{Si}$ interstitials compared to $\mathrm{C}$ interstitials (Table 3). A more important observation is that the swelling seems to linearly depend on Si and C FP. Moreover, the effect of Si and C sub-lattice defects is apparently not related, or only weakly. However, there is a large swelling reduction for the HT simulations, that suggests an important modification of the defects distributions, like a decrease due to recombination for instance, or a transformation through processes like aggregations.

To gain further insights, a deeper investigation is required. A Wigner-Seitz cell analysis is then performed to determine the defects distribution for each calculated systems reported in Fig. 2. Figure 3 represents the final amounts of point defects relative to the initial numbers of $\mathrm{C}$ or $\mathrm{Si} \mathrm{FP}$, averaged over all configurations. First, there is a significant decrease of $\mathrm{I}_{\mathrm{Si}}$ at RT (about 18\%). This effect is more important at HT, with only about $17 \%$ in average remaining at the end of the simulations. Conversely, the amount of $\mathrm{I}_{\mathrm{C}}$ barely decreases at $\mathrm{RT}$, and a significant creation of new defects is observed for HT simulations, with a $21 \%$ increase. It is instructive to correlate this information with the variation of $\mathrm{Si}_{\mathrm{C}}$ antisites. In fact, for RT simulations, about $15 \%$ are created (relative to the initial Si FP), and for HT ones, approximately $112 \% \mathrm{Si}_{\mathrm{C}}$ are created. These results can be easily explained by the following transformation

$$
\mathrm{I}_{\mathrm{Si}} \longrightarrow \mathrm{I}_{\mathrm{C}}+\mathrm{Si}_{\mathrm{C}}
$$

The potential from Erhart and Albe tends to underestimate the formation energy of $\mathrm{Si}_{\mathrm{C}}$, while overestimating the one of $\mathrm{I}_{\mathrm{Si}}$ [13]. As a consequence the reaction (7) is energetically favored with a likely too large energy gain. It is then probably

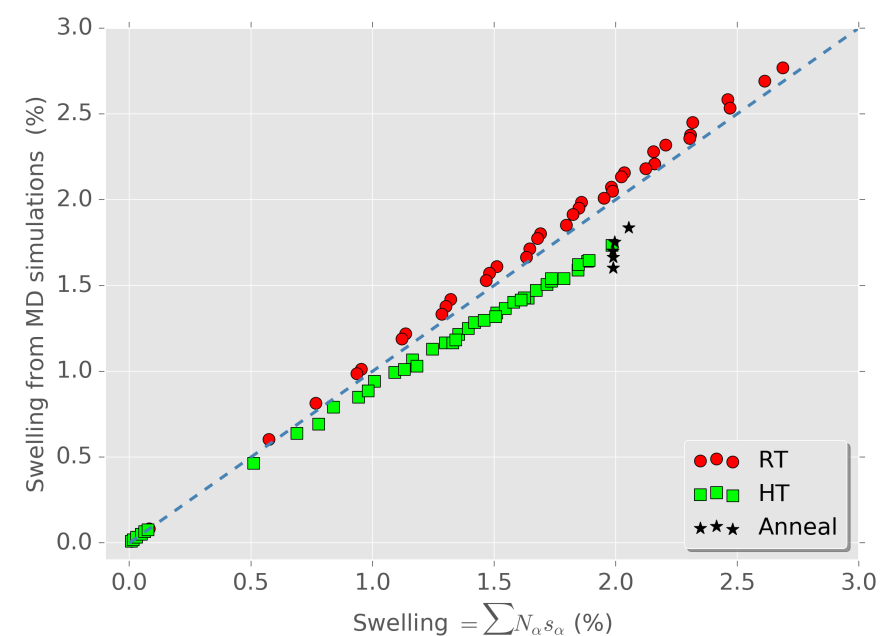

Figure 4: Swelling in large MD simulations versus swelling estimated from the sum of relaxation volumes of all point defects, determined at $300 \mathrm{~K}$. The dashed blue lines marks the equality between these two quantities. Each red circle (green square) represent one RT (HT) MD simulations. The black stars represent simulations at different annealing temperatures, described in section 5 .

not physically realistic, but its effect on the swelling appears fortunately to be rather limited. In fact, the average relaxation volume for $\mathrm{I}_{\mathrm{Si}}$ is approximately equal to the sum of the relaxation volumes of $\mathrm{Si}_{\mathrm{C}}$ and $\mathrm{I}_{\mathrm{C}}$ (table 3 ), suggesting an equivalent contribution to the total swelling.

Concerning $\mathrm{V}_{\mathrm{Si}}$ and $\mathrm{V}_{\mathrm{C}}$, a small decrease is obtained in $\mathrm{RT}$ simulations, with more than $90 \%$ vacancies present at the end of the runs. In the case of HT simulations, the decrease is more pronounced. In both cases, carbon vacancies are more prone to disappear than silicon vacancies. Finally, almost no $\mathrm{C}_{\mathrm{Si}}$ antisite defects form during RT simulations, but their proportion rises to $22 \%$ at the end of HT runs. Note that in most cases the standard deviations, also reported in Fig. 3, are small, especially for RT simulations. This reflects the fact that the final amounts of point defects are proportional to the initial numbers of $\mathrm{C}$ or Si FP, and validates the choice to consider averages for analyzing defects distributions. Significant exceptions are the standard deviations for $\mathrm{Si}_{\mathrm{C}}$ and $\mathrm{I}_{\mathrm{C}}$ at $\mathrm{HT}$, because they are normalized by either the initial numbers of $\mathrm{Si} \mathrm{FP}$ (for $\mathrm{Si}_{\mathrm{C}}$ ) or $\mathrm{C} \mathrm{FP}$ (for $\mathrm{I}_{\mathrm{C}}$ ), although they depend on both through the relation (7).

The Wigner-Seitz cell analysis also reveals the presence of defects more complex than single $\mathrm{C}$ or $\mathrm{Si}$ interstitials. For instance, C-C ( $\mathrm{Si}-\mathrm{Si})$ dumbbells located in a $\mathrm{Si}(\mathrm{C})$ site are frequently obtained. Small aggregates are also possible, such as $\mathrm{Si}_{3}, \mathrm{Si}_{4}, \mathrm{C}_{3}$, or $\mathrm{Si}_{2} \mathrm{C}, \mathrm{SiC}_{2}$, etc... Their occurrences are greater in HT than in RT conditions, the high temperature allowing for an enhanced migration and interaction between interstitials. However, their proportion relative to the total amount of point defects remains small. For instance, considering an initial configuration including $1000 \mathrm{Si}$ and C FP, one finds 5 complex defects at RT, and 38 at HT. This has to be compared to a total number of 3639 (3415) single point defects at the end of RT (HT) simulations. One interesting point is that these complex defects are typically positioned in C sites in RT simulations, 


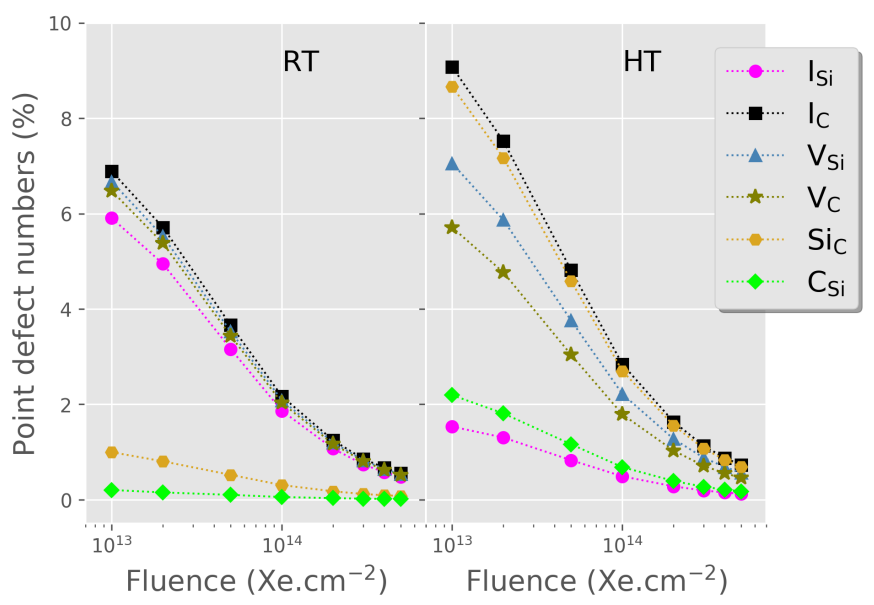

Figure 5: Point defects numbers for different fluences in both RT and HT conditions, estimated so as to yield the same swelling than in experiments (reported in Fig. 1). Values are expressed as percentages, relative to the numbers of Si FP (for $\mathrm{I}_{\mathrm{Si}}, \mathrm{V}_{\mathrm{Si}}$, and $\mathrm{Si}_{\mathrm{C}}$ ) and of $\mathrm{C} \mathrm{FP}$ (for $\mathrm{I}_{\mathrm{C}}, \mathrm{V}_{\mathrm{C}}$, and $\mathrm{C}_{\mathrm{Si}}$ ) as yielded by SRIM calculations (see text for details).

and in Si sites in HT simulations. Yet it is difficult to explain this observation.

Now that point defect distributions are known for all cases, it is possible to compare the swelling at the end of the simulations with the approximate value $\sum_{\alpha} N_{\alpha} s_{\alpha}, N_{\alpha}$ being the amount of point defect of type $\alpha$. Figure 4 shows a good agreement between both approaches for RT conditions, albeit the summation slightly underestimates the MD swelling. It is possible that the difference is caused by the low temperature and short simulation time, that does not allow for a full relaxation of the random initial defect distribution during RT runs. In the case of HT conditions, I observe that the summation yields swelling value greater than in simulations, the difference increasing with the defects concentration. It is also noteworthy that this discrepancy is in fact underestimated because only single point defects are considered in the summation, that does not include the previously described complexes. Although it is difficult to be definite on this point, it is likely that the difference in HT conditions could be explained by a local restructuration during the high temperature calculations. In fact, close point defects can organize amongst themselves as to minimize elastic interactions, thus decreasing the overall relaxation volume. Nevertheless, I note that the discrepancy is at most of $15 \%$, which suggests that using the summation approach is a good approximation if the defects distribution is known.

At last, I try to compare SRIM calculations with possible defects distributions in agreement with swelling measurements. Reference SRIM calculations are first performed for a $540 \mathrm{keV}$ Xe implantation in a $500 \mathrm{~nm} \mathrm{SiC} \mathrm{slab,} \mathrm{using} \mathrm{threshold} \mathrm{dis-}$ placement energies of $20 \mathrm{eV}$ and $35 \mathrm{eV}$ for $\mathrm{C}$ and $\mathrm{Si}$, respectively [27]. In a second step, I define a function of three variables, $N_{\mathrm{Si}}, N_{\mathrm{C}}$, and $N_{\mathrm{Xe}}$, i.e. the numbers of $\mathrm{Si}$ and $\mathrm{C} \mathrm{FP}$, and of implanted Xe atoms. For any values of these three variables, one can obtain the amounts of each kind of single point defects $N_{\alpha}$ by linear interpolation, using either RT or HT sets of sim-

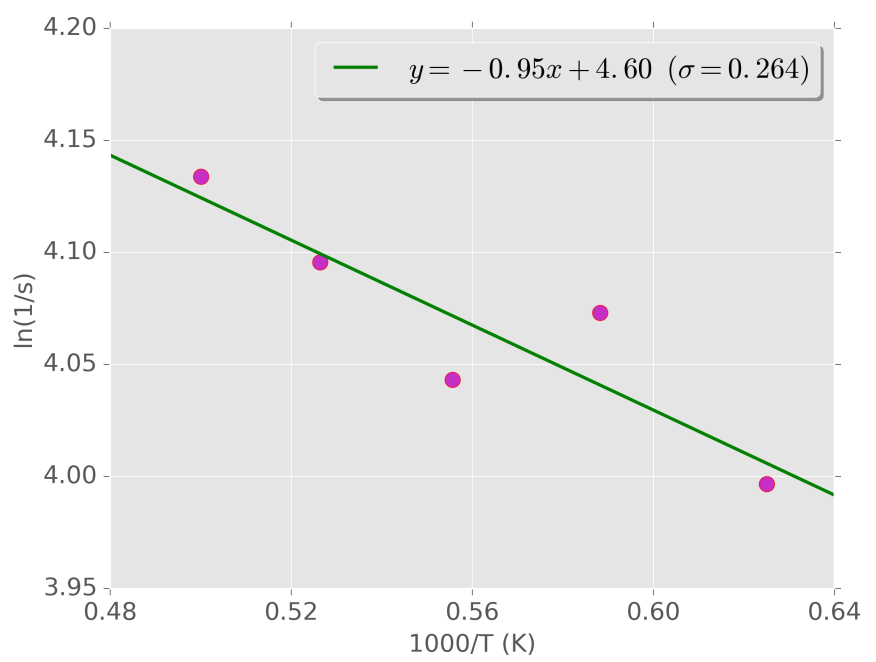

Figure 6: Computed swelling after $1 \mathrm{~ns}$ annealing as a function of temperature (magenta dots). The linear regression through these points is plotted as a green line. The slope coefficient corresponds to an activation energy of $82 \mathrm{meV}$.

ulations. Furthermore, using the relaxation volumes reported in Table 3, a unique swelling value $s$ can be computed. Next I aim at determining $N_{\mathrm{Si}}, N_{\mathrm{C}}$, and $N_{\mathrm{Xe}}$ such that $s$ is equal to the experimental swelling. To reduce the number of possible solutions to only one, I use the fact that the number of implanted $\mathrm{Xe}$ atoms is known and I assume that the ratio between $N_{\mathrm{Si}}$ and $N_{\mathrm{C}}$ is constant, like in SRIM calculations. Then only one variable needs to be adjusted to obtain the best agreement between computed and measured swelling, with a unique solution. This procedure is numerically performed for each swelling measurements, and the resulting defects distributions for different fluences are determined for both RT and HT conditions. The latter are shown in Fig. 5, as percentages of the SRIM predicted amounts of C and SI FP. Those, computed using the Kinchin-Pease model, correspond to concentrations ranging from $1.25 \times 10^{21}{\mathrm{FP} . \mathrm{cm}^{-3}}^{-3}$ to $6.24 \times 10^{22} \mathrm{FP}_{\mathrm{cm}}{ }^{-3}$ for the Si sub-lattice $\left(1.88 \times 10^{21} \mathrm{FP} \mathrm{cm}^{-3}\right.$ to $9.39 \times 10^{22} \mathrm{FP}^{-\mathrm{cm}^{-3}}$ for the $\mathrm{C}$ sub-lattice) for a fluence in the range $1 \times 10^{13}-5 \times$ $10^{14} \mathrm{Xe} . \mathrm{cm}^{-2}$. The most prominent differences between RT and HT setups concern the strong reduction of $\mathrm{Si}$ interstitials leading to a high proportion of $\mathrm{I}_{\mathrm{C}}$ and $\mathrm{Si}_{\mathrm{C}}$ through the relation (7), together with the largest amount of $\mathrm{C}_{\mathrm{Si}}$ in HT conditions. However, one can see that at the lowest fluences the vacancies and interstitials concentrations would be 6-9\% of the SRIM predictions in both cases. SRIM calculations become increasingly inaccurate with the fluence, with final proportions lower than $1 \%$ of the predictions in both RT and HT conditions.

\section{Evolution during annealing}

Previous experiments have shown that the maximum swelling associated with xenon implantation decreases with the temperature according to an Arrhenius-like behavior, and activation energies in the range [67-70] meV $[10,28]$. In this section the relation between swelling and temperature is investigated, using the large systems described above. Since human timescales 


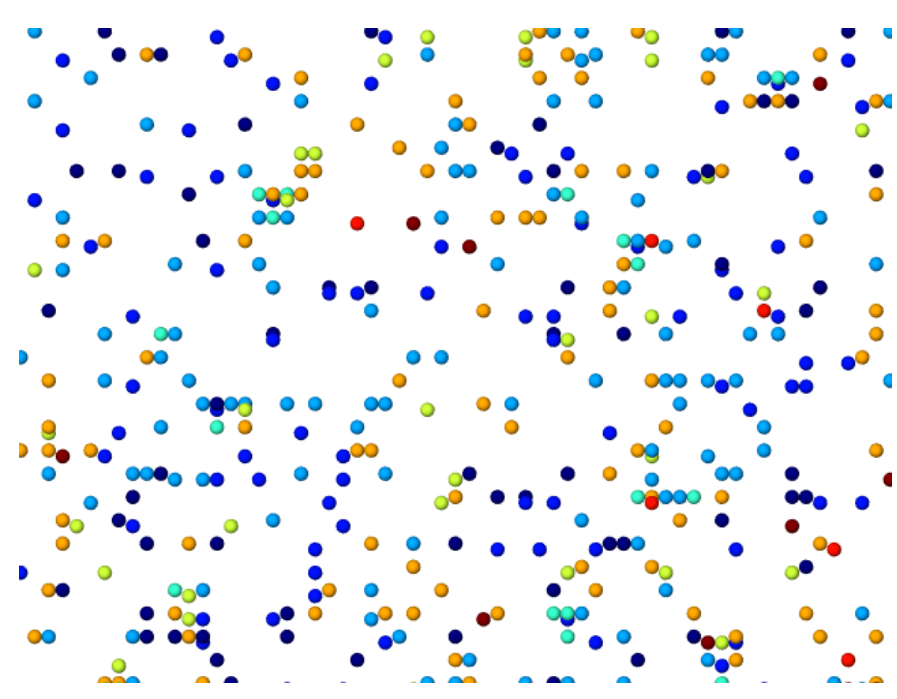

Figure 7: Spatial distribution of point defects and Xe atoms at the end of the $2000 \mathrm{~K}$ annealing. Spheres represent the lattice sites with a Wigner-Seitz cell occupancy different than in a perfect $\mathrm{SiC}$ lattice. The sphere color corresponds to the kind of defects: $\mathrm{V}_{\mathrm{C}}$ (black), $\mathrm{V}_{\mathrm{Si}}$ (dark blue), $\mathrm{I}_{\mathrm{C}}$ (light blue), $\mathrm{I}_{\mathrm{Si}}$ (cyan), $\mathrm{C}_{\mathrm{Si}}$ (green), $\mathrm{Si}_{\mathrm{C}}$ (orange), $\mathrm{Xe}$ (red), small aggregates (brown). Only point defects in a $20 \AA$ thick slice are shown for clarity.

are out of the reach of molecular dynamics, temperatures higher than in Ref. [10] are used, from $1600 \mathrm{~K}$ to $2000 \mathrm{~K}$ by steps of $100 \mathrm{~K}$, in order to enhance mechanisms at work. Obviously such an approach relies on the assumption that similar mechanisms are activated in simulations and in experiments at lower temperatures.

Only one initial point defect configuration, including $60 \mathrm{I}_{\mathrm{Xe}}$, $1000 \mathrm{C} \mathrm{FP}$, and $1000 \mathrm{Si} \mathrm{FP}$, is used in these MD simulations. As previously, a 0.1 ps run is first performed in the NVE ensemble with maximum atomic displacements set to $0.01 \AA$ at each step. Then a 1 ps run is done at the chosen temperature to equilibrate the system. Finally, the system evolve at the same temperature during $10 \mathrm{~ns}$, with allowed supercell expansion along $\vec{c}$.

For each temperature, the swelling is seen to continuously decrease during the MD calculations, rather sharply at the beginning and with a low rate at the end. In addition, the final swelling value significantly depends on the simulation temperature. In Fig. 6, the logarithm of the inverse of the swelling value is represented versus the inverse of the temperature. A linear regression through the calculated data yields an activation energy of $82 \mathrm{meV}$, in excellent agreement with the experiments $[10,28]$. Although it is possible that the MD simulations effectively reproduce the same mechanisms than in experiments, one has to be cautious that such an agreement might well be fortuitous. In fact, in experiments, the swelling decrease is thought to be due to dynamic recombination during implantation. This has to be compared to simulations, initiated with a spatially homogeneous distribution of defects, and with temperature only to activate mechanisms.

To identify the mechanisms decreasing the swelling, on can gain further insights by analysing a possible point defects organization. Figure 7 shows the spatial distribution of point defects at the end of the $2000 \mathrm{~K}$ MD simulation. Obviously there are

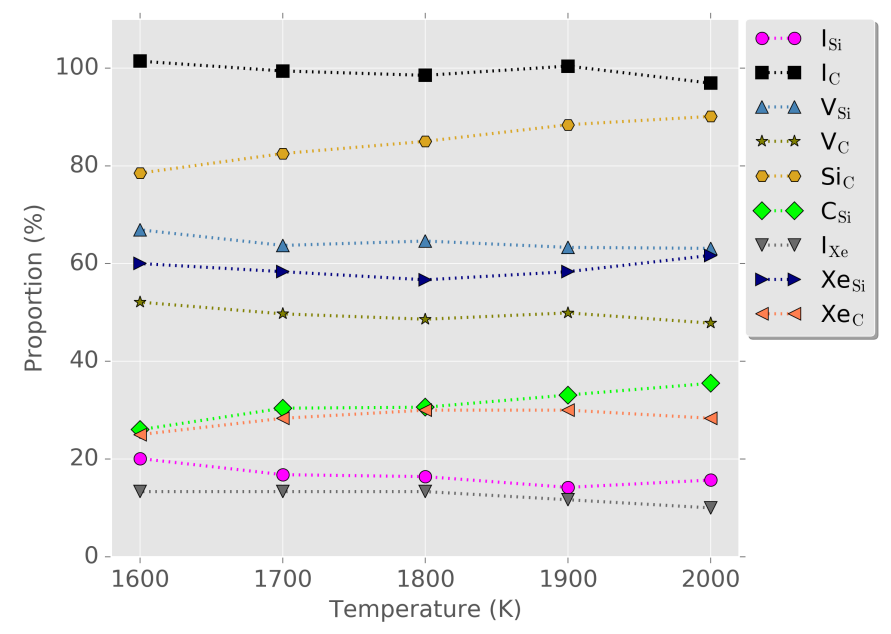

Figure 8: Proportion (in \%) of point defects at the end of long MD simulations, as a function of temperature, relative to the initial amount of Si/C FP (1000) or $\mathrm{Xe}(60)$ : $\mathrm{I}_{\mathrm{Si}}$ (pink circles), $\mathrm{I}_{\mathrm{C}}$ (black squares), $\mathrm{V}_{\mathrm{Si}}$ (steel blue triangles up), $\mathrm{V}_{\mathrm{C}}$ (olive stars), $\mathrm{Si}_{\mathrm{C}}$ (yellow hexagons), $\mathrm{C}_{\mathrm{Si}}$ (dark blue diamonds), $\mathrm{I}_{\mathrm{Xe}}$ (grey triangles down), $\mathrm{Xe}_{\mathrm{Si}}$ (green triangles right), $\mathrm{Xe}_{\mathrm{C}}$ (orange triangles left).

no evidences of point defects aggregation, such as clusters of interstitials or vacancies. This suggests that the decrease of the swelling is not due to point defects clustering, but more likely to a decrease of the amount of point defects through recombination. To better support this idea, I plot the variation of point defects concentrations for each species as a function of temperature in Fig. 8. First I note that the different point defects quantities are relatively similar to those reported at the end of short HT MD runs, hinting that point defects evolution mechanisms are the same. In fact, I observe the formation of $\mathrm{Si}_{\mathrm{C}}$ antisites through relation (7) and xenon interstitials becoming progressively positioned in $\mathrm{Si}$ or $\mathrm{C}$ vacancies, in agreement with experiments [29]. Then the most prominent change as a function of temperature, with respect to swelling reduction, is the decrease of $\mathrm{I}_{\mathrm{Si}}, \mathrm{V}_{\mathrm{Si}}$, and $\mathrm{V}_{\mathrm{C}}$, and the increase of $\mathrm{Si}_{\mathrm{C}}$ and $\mathrm{C}_{\mathrm{Si}}$. This is compatible with the recombination of interstitials with vacancies, growing with temperature. According to previous theoretical studies the more mobile species are $\mathrm{C}$ and $\mathrm{Si}$ interstitials, with low calculated migration energies [21] in agreement with the calculated activation energy of $82 \mathrm{meV}$. This work then confirms that the swelling reduction as a function of temperature is certainly due to the recombination of point defects by the migration of interstitial species.

\section{Conclusions}

To conclude, the contribution of point defects to strain build up in $4 \mathrm{H}-\mathrm{SiC}$ has been determined using atomistic calculations, in relation with Xe implantation experiments. The relaxation volume and the associated strain are computed for various point defects, within stress conditions similar than in experiments. These simulations first confirm that heavy noble gas species like xenon have a negligible contribution to strain build up and to the resulting swelling. Another interesting result is that the total strain can be correctly estimated from the summation of 
single defect contributions. By comparing experimental and calculated swelling data, the distribution of point defects as a function of dose is also determined. Finally, annealing simulations reveal a reduction of swelling due to dynamic recombination of single point defects, with an activation energy in excellent agreement with experiments.

In the present work, an initial homogeneous distribution of point defects is chosen as representative of a post-implanted material. This is obviously oversimplified, but convenient and reasonable as a first try to understand the relation between point defects and swelling. Nevertheless, the true implanted state is certainly more complex. For instance, the observation of black spot defects in microscopy experiments suggests that a non negligible proportion of point defects is aggregated [24]. Such extended structures are difficult to obtain in the course of MD simulations due to the time scale limitation of this technique, and should be introduced in the initial state of the simulation. This leaves room for future investigations.

\section{Acknowledgements}

This work has been financially supported by the Défi NEEDS Matériaux program. Emmanuel clouet is acknowledged for fruitful discussions concerning the elastic description of point defects, and Aurélien Debelle for the critical reading of the manuscript The author is also indebted to Prof. Jean-François Barbot for valuable comments about the manuscript and for providing experimental data.

\section{References}

[1] G. Harris (Ed.), Properties of silicon carbide, INSPEC, Institution of Electrical Engineers, London, 1995.

[2] W. J. Choyke, G. Pensl, Physical properties of sic, Mater. Res. Soc. Bull. 22 (3) (1997) 25.

[3] Y. Katoh, L. Snead, C. H. Jr., A. Hasegawa, A. Kohyama, B. Riccardi, H. Hegeman, Current status and critical issues for development of sic composites for fusion applications, Journal of Nuclear Materials 367370, Part A (2007) 659-671.

[4] T. Hinoki, Y. Katoh, L. Snead, H.-C. Jung, K. Ozawa, H. Katsui, Z.-H. Zhong, S. Kondo, Y.-H. Park, C. Shih, C. Parish, R. Meisner, A. Hasegawa, Silicon carbide and silicon carbide composites for fusion reactor application, Materials Transactions 54 (4) (2013) 472.

[5] W. J. Weber, W. Jiang, S. Thevuthasan, Accumulation, dynamic annealing and thermal recovery of ion-beam-induced disorder in silicon carbide, Nucl. Instrum. Methods Phys. Res. B 175-177 (2001) 26.

[6] F. Gao, W. J. Weber, Atomic-level computer simulation of SiC: defect accumulation, mechanical properties and defect recovery, Philos. Mag. 85 (4-7) (2005) 509-518. doi:10.1080/02678370412331320170.

[7] A. Debelle, A. Boulle, A. Chartier, F. Gao, W. J. Weber, Interplay between atomic disorder, lattice swelling, and defect energy in ionirradiation-induced amorphization of sic, Phys. Rev. B 90 (2014) 174112. doi:10.1103/PhysRevB.90.174112.

[8] S. Leclerc, A. Declmy, M. F. Beaufort, C. Tromas, J. F. Barbot, Swelling of sic under helium implantation, Journal of Applied Physics 98 (11) (2005) 113506. doi:10.1063/1.2137441.

[9] S. Leclerc, M. F. Beaufort, A. Declmy, J. F. Barbot, Evolution of defects upon annealing in he-implanted 4h-sic, Applied Physics Letters 93 (12) (2008) 122101. doi:10.1063/1.2988262.

[10] C. Jiang, J. Nicola, A. Declémy, E. Gilabert, M.-F. Beaufort, J.-F. Barbot, Implantation damage in heavy gas implanted $4 \mathrm{~h}$-sic, Nucl. Instrum. Meth. B 374 (2016) 71.
[11] J. Tersoff, Modeling solid-state chemistry: Interatomic potentials for multicomponent systems, Phys. Rev. B 39 (8) (1989) 5566.

[12] F. Gao, W. Weber, Empirical potential approach for defect properties in 3c-sic, Nucl. Instrum. Meth. B 191 (2002) 504.

[13] P. Erhart, K. Albe, Analytical potential for atomistic simulations of silicon, carbon, and silicon carbide, Phys. Rev. B 71 (2005) 035211.

[14] G. Lucas, M. Bertolus, L. Pizzagalli, An environment-dependent interatomic potential for silicon carbide: calculation of bulk properties, highpressure phases, point and extended defects, and amorphous structures, J. Phys.: Condens. Matter 22 (2010) 035802.

[15] M. Bertolus, Modélisation à l'échelle atomique de matériaux nucléaires du cycle du combustible, Report, CEA (2011).

[16] A. Charaf Eddin, L. Pizzagalli, First principles calculation of noble gas atoms properties in 3csic, J. Nucl. Mater. 429 (13) (2012) 329 - 334. doi:http://dx.doi.org/10.1016/j.jnucmat.2012.06.022.

[17] J. F. Ziegler, U. Littmark, J. P. Biersack, The stopping and range of ions in solids, Pergamon New York, 1985.

[18] A. K. Dham, W. J. Meath, A. Allnatt, R. A. Aziz, M. Slaman, Xc and hfd-b potential energy curves for xe-xe and related physical properties, Chemical Physics 142 (1990) 173.

[19] S. Plimpton, Fast parallel algorithms for short-range molecular dynamics, J. Comput. Phys. 117 (1) (1995) $1-19$.

[20] F. Gao, W. J. Weber, Recovery of close frenkel pairs produced by low energy recoils in sic, J. Appl. Phys. 94 (7) (2003) 4348.

[21] M. Bockstedte, A. Mattausch, O. Pankratov, Ab initio study of the annealing of vacancies and interstitials in cubic sic: Vacancy-interstitial recombination and aggregation of carbon interstitials, Phys. Rev. B 69 (2004) 235202.

[22] L. Torpo, M. Marlo, T. E. M. Staab, R. M. Nieminen, Comprehensive ab initio study of properties of monovacancies and antisites in $4 \mathrm{~h}$-sic, J. Phys.: Condens. Matter 13 (28) (2001) 6203.

[23] J. Li, L. Porter, S. Yip, Atomistic modeling of finite-temperature properties of crystalline -sic: Ii. thermal conductivity and effects of point defects, Journal of Nuclear Materials 255 (2) (1998) 139 - 152. doi:https://doi.org/10.1016/S0022-3115(98)00034-8.

[24] B. Tyburska-Püschel, Y. Zhai, L. He, C. Liu, A. Boulle, P. Voyles, I. Szlufarska, K. Sridharan, Size distribution of black spot defects and their contribution to swelling in irradiated SiC, J. Nucl. Mater. 476 (2016) 132139. doi:10.1016/j.jnucmat.2016.04.044.

[25] A. Debelle, A. Declémy, XRD investigation of the strain/stress state of ion-irradiated crystals, Nuclear Instruments and Methods in Physics Research Section B: Beam Interactions with Materials and Atoms 268 (9) (2010) 1460-1465. doi:10.1016/j.nimb.2010.01.009.

[26] J. F. Ziegler, M. Ziegler, J. Biersack, \{SRIM\} the stopping and range of ions in matter (2010), Nuclear Instruments and Methods in Physics Research Section B: Beam Interactions with Materials and Atoms 268 (2010) $1818-1823$.

[27] G. Lucas, L. Pizzagalli, Ab initio molecular dynamics calculations of threshold displacement energies in silicon carbide, Phys. Rev. B 72 (2005) $161202 R$.

[28] J. Li, C. Zhang, C. Xu, X. Jia, Y. Song, J. Li, Y. Jin, Lattice damage and nanohardness in 6hsic implanted with multiple-energy xe ions, Nuclear Instruments and Methods in Physics Research Section B: Beam Interactions with Materials and Atoms 286 (2012) $124-128$, proceedings of the Sixteenth International Conference on Radiation Effects in Insulators (REI). doi:https://doi.org/10.1016/j.nimb.2011.11.034.

[29] C. Zhang, Y. Sun, Y. Song, T. Shibayama, Y. Jin, L. Zhou, Defect production in silicon carbide irradiated with ne and xe ions with energy of 2.3 mev/amu, Nucl. Instrum. Methods Phys. Res. B 256 (2007) 243. 